\title{
Evaluación de la actividad alelopática de extractos crudos de Copaifera pubiflora (Benth), sobre la germinación de Mimosa pudica (Lineo)
}

\author{
José Luis Rodríguez-Gutiérrez, Lady Johana Correa-Higuera, \\ Andrés Enrique Alvarado-Camacho*, Jorge Alberto Chaparro-Pesca \\ Fundación Universitaria Internacional del Trópico Americano, Yopal, Casanare, Colombia
}

\begin{abstract}
Resumen
Los efectos alelopáticos son una herramienta para el control de arvenses más práctica y viable en los agroecosistemas, estas reacciones naturales tienen múltiples efectos, que van desde la inhibición o estimulación de los procesos de crecimiento de las plantas vecinas, hasta la inhibición de la germinación de semillas. En este trabajo se evaluó la actividad alelopática de extractos brutos de Copaifera pubiflora (Benth) sobre la germinación de Mimosa pudica (Lineo) para generar nuevos posibles métodos de control de $M$. pudica, y al mismo tiempo ampliar el conocimiento y bioprospección de especies endémicas de la Orinoquia, como lo es C. pubiflora. Se utilizaron extractos etanólico, hexánico y medio acuoso, de hojas y corteza de C. pubiflora, en concentraciones de 7, 5 y $2 \%$ pv y un diseño multi-factor categórico, junto con pruebas preliminares de identificación de metabolitos secundarios para el extracto con mayor actividad alelopática. Los resultados mostraron que los extractos etanólico y en medio acuoso de corteza y hojas, tienen actividad alelopática, sobre la germinación de M. pudica. Además, con las pruebas preliminares se logró determinar la posible presencia de metabolitos secundarios que son reconocidos por generar alelopatía. (C) 2016. Acad. Colomb. Cienc. Ex. Fis. Nat.
\end{abstract}

Palabras clave: Alelopatía; Inhibición de germinación; Extractos crudos; C. pubiflora; M. pudica.

Allelopathic activity evaluation of Copaifera pubiflora (Benth), crude extracts on the germination of Mimosa pudica (Linnaeus)

\begin{abstract}
The allelopathic effects are a tool for the control of viable arvenses in the agro ecosystems, these natural reactions have multiple effects that go from the inhibition or stimulation of the neighbour plants growth until the inhibition of the seeds germination. In this work, the allelopathic activity of crude extracts of Copaifera pubiflora (Benth) on the germination of Mimosa pudica (Lineo) was evaluated to generate new potential control methods of $M$. pudica, and knowledge of endemic species of the Orinoco, as C. pubiflora and their potential uses. Ethanolic, hexane and aqueous extracts 7.5 and $2 \% \mathrm{wv}$ of leaves and bark of $C$. pubiflora were used. A multi-factor categorical design and preliminary identification tests of secondary metabolites were performed. The results showed that ethanolic and aqueous extracts of bark and leaves have allelopathic activity on the germination of $M$. pudica. In addition, preliminary tests of secondary metabolites determined the possible presence of some family compounds which are recognized by their allelopathic activity. (C) 2016. Acad. Colomb. Cienc. Ex. Fis. Nat.
\end{abstract}

Key words: Allelopathy; Inhibition of germination; Crude extracts; C. pubiflora; M. pudica.

\section{Introducción}

La creciente demanda de alimentos, ha propiciado la adopción de sistemas de producción en masa, los cuales buscan mayor eficiencia del uso del suelo pero su manejo inadecuado limita su capacidad productiva y altera su desempeño agrícola. Para lograr este objetivo se requiere de numerosas entradas de agroquímicos al agro-ecosistema, pero con el tiempo esto produce baja fertilidad del suelo, disminución de la diversidad biológica y por consiguiente contaminación del medio ambiente, así mismo los arvenses pueden generar resistencia a los herbicidas como lo señala
Menza \& Salazar, 2006, en consecuencia la toxicidad y cantidad de los compuestos se debe aumentar ciclo tras ciclo, potenciando los efectos negativos lo que conlleva a la degradación del ecosistema de forma exponencial.

En este sentido, la característica estacionaria de las plantas, ha hecho que desarrollen diferentes mecanismos de comunicación y defensa. Uno de los más importantes

\footnotetext{
*Correspondencia:

Andrés Enrique Alvarado-Camacho, andresalvarado@unitropico.edu.co Recibido: 24 de mayo de 2016

Aceptado: 24 de agosto de 2016
} 
es el mecanismo químico, en donde las plantas producen metabolitos secundarios y los liberan al medio; dentro de ellos los aleloquímicos, causantes del fenómeno conocido como alelopatía química, son los que especialmente han venido siendo estudiados para desarrollar controladores naturales de arvenses o plagas, (Oliveros, 2008), sin embargo su efecto puede ser muy específico sobre una o algunas especies, lo que impide generalizar un compuesto para un grupo grande de plantas (Blanco, 2013).

El uso desmedido de agroquímicos, en los sistemas productivos, ha contribuido a la degradación del ecosistema; en los últimos años esto ha generado preocupación en la comunidad internacional, por lo que el desarrollo de nuevas estrategias de control como el uso de la alelopatía se han convertido en una opción potencialmente viable según Zamorano, 2006, en este sentido la alelopatía se define según la Sociedad de Alelopatía Internacional, 1996 "Como el proceso en el que una planta desprende al medio ambiente, uno o varios, compuestos químicos que inhiben el crecimiento de otra planta que vive en el mismo hábitat o en un hábitat cercano" y de igual manera Narwal \& Haouala, 2011, la definen como "el proceso natural que envuelve la producción de un metabolito secundario (aleloquímico) por las plantas, que una vez liberado al ambiente influye sobre el crecimiento y desarrollo de sistemas agrícolas". Por otra parte se ha registrado el efecto inhibitorio sobre la germinación de semillas a causa de los compuestos exudados tanto por especies cultivadas como por ciertas especies no cultivadas entre las que se incluyen las malezas según Mehhboob, Saleem, y Qureshi, 2000 Es así que el desarrollo de esta investigación contribuye a generar una nueva opción de control de $M$. pudica (Lineo) considera un arvense en los sistemas productivos.

Mimosa pudica (Dormidera), vergonzosa o dormidera (M. pudica) es una planta de origen americano de la familia de las fabáceas, fácilmente distinguible por su reacción al tacto, desarrollada como defensa ante los predadores, (Bernal, Martínez y Sánchez, 2011). M. pudica contienen un alcaloide tóxico llamado mimosina, que tiene efectos anti proliferativos y genera apoptosis. Los extractos también inmovilizan las larvas de Strongyloides stercoralis en menos de una hora, y además los extractos acuosos de las raíces de la planta han mostrado efectos neutralizantes frente a la letalidad del veneno de cobra, donde parece inhibir su toxicidad y actividad enzimática (Cuite, Rosales y Gámez, 2015).

Existen impactos agrícolas considerables en algunos cultivos propios de las zonas tropicales como los cultivos de cacao, caña de azúcar, cocoteros, palma de aceite, café, banano, arroz y piña como lo indican (Abad, Tiscareño, Villar y Varela, 2012). Ariza \& Merchán, 2012 reportan esta especie como una arvense asociada a los cultivos de palma de cera en Casanare y Meta.

Copaifera pubiflora (Benth), es una especie endémica de Colombia, particularmente de la Orinoquia (Bernal, et al. 2011) aunque se tienen algunos registros sobre el uso medicinal y culinario del aceite (Acero, 2005) otros usos potenciales como controlador de arvenses son desconocidos; esta descrito como un un árbol llamativo por su copa, su follaje y el color anaranjado de su corteza, presenta exudación aceitosa extraída mediante taladro o perforación en el fuste y normalmente es usada como antitetánico aplicando en la herida o también para ahuyentar las moscas en el ganado (Acero, 2005). El género Copaifera ha sido poco estudiado en Colombia, pero presenta publicaciones donde referencian tres especies nativas del Neo trópico y una especie endémica de la Orinoquia, que es el árbol de aceite (C. pubiflora), con cuatro reportes de uso, al igual que la copaiba (C. canime), con cuatro registros (Bernal, et al. 2011), por otra parte también (C. langsdorffii) ha demostrado efectos inhibitorios en el crecimiento de las raíces, (Franco, 2013), también se han hecho estudios de dos especies emparentadas $C$. duckei y C. reticulata que demuestran actividad alelopática en aceite esencial de las hojas y el tallo (Gurgel, 2009); Asi mismo, según un estudio de Ramírez y Arroyo, (1990) C. pubiflora tiene una estructura poblacional determinada por la edad, las plantas menores a 50 años muestran una tendencia pronunciada y acentuadamente en las sabanas de la Orinoquia, mientras que a los 25 años hay ausencia de individuos y posterior a este hay un ligero incremento poblacional; la ausencia de publicaciones se deba a que ha sido confundido con especies del mismo género de origen amazónico; en este caso el género con más publicaciones es Copaifera $L$. que pertenece a la familia de las Fabaceae, que tiene cerca de 727 géneros y 19.325 especies (Lewis, et al. 2005). Los extractos de este género han sido usados por algunas comunidades indígenas de la amazonia y los llamaron el "producto milagro" (Ferreira, 1988); (Cunha, 1999); (Martinsda-Silva, 2006).

La región amazónica es probablemente el principal proveedor de esta oleorresina utilizada en los mercados brasileros y del mundo (Cascón \& Gilbert, 2000). Este aceite posee propiedades antiinflamatorias y es usado en la medicina popular, (Martins-da-Silva, 2006). Por lo tanto, es evidente la escasa investigación que se tiene del genero Copaifera pubiflora en comparación con los géneros ya mencionados de la amazonia; el desarrollo de investigaciones sobre esta llevara a conocer sobre sus características y desempeño en los diferentes agro-ecosistemas y de esta manera contribuirá a generar conocimiento de la diversidad biológica de la Orinoquia; de esta manera será probable usar este conocimiento en agroforesteria en el conocimiento de las interacciones biológicas entre especies, con el objetivo de utilizarlas para beneficio del sistema agroforestal, como lo indican Ospina, et al. (2003), señalan que la mayoría de los estudios en alelopatía van dirigidos a incluirla en los sistemas de producción para aumentar la productividad de los cultivos, en este trabajo se presenta un aporte primario para conocer las interacciones entre C. pubiflora y M. pudica.

Blanco, (2006) establece que la utilidad de la alelopatía es una herramienta más que puede contribuir a generar cultivos verdes y que estos efectos alelopáticos son una herramienta de manejo en los cultivos que pueden ser de los 
usos más prácticos y aplicables en los agro ecosistemas, estas reacciones naturales tienen múltiples efectos, que van desde la inhibición o estimulación de los procesos de crecimiento de las plantas vecinas, hasta la inhibición de la germinación de semillas. Así mismo, los efectos alelopáticos que dañan parcial o totalmente la germinación y el crecimiento de las plantas, pueden ser de carácter positivo o negativo, directo e indirecto, según la concentración de las sustancias y el tiempo de aplicación de la concentración empleada.

\section{Material y métodos}

Ubicación del ensayo. La recolección del material vegetal se realizó en el área rural del municipio de Yopal-Casanare, los árboles de C. pubiflora se ubicaron en las siguientes coordenadas N 5'22'16.3164", W 72²1'12.4812" y N $5^{\circ} 26^{\prime} 17.8296^{\prime \prime}, \mathrm{W} 72^{\circ} 16^{\prime} 3.3492$ ', mientras las semillas de M. púdica se recolectaron de plantas en las coordenadas N 5²0'15.7621”, W 72²2’36.7612” (World Geodesic System, 1984). Los bioensayos se desarrollaron en el laboratorio de fitoquímica de la Fundación Universitaria Internacional del Trópico Americano, Unitrópico

Material vegetal. Se recolectaron hojas y corteza de $C$. pubiflora, de árboles que tenían un buen porte, completamente desarrolladas y en buen estado fitosanitario. Las semillas de $M$. pudica, se recolectaron y seleccionaron utilizando juego de tamices de $1,7 \mathrm{~mm}$ a $2 \mathrm{~mm}$, a continuación se sumergieron en agua, con el objetivo de seleccionar las más viables para germinación y al mismo tiempo homogenizarlas (Labouriao, 1983).

Obtención de extractos crudos. Los extractos crudos fueron obtenidos a partir de hojas y tallos de C. pubiflora por separado utilizando hexano, etanol y agua como solventes.

El material vegetal (hojas y tallo de C. pubiflora) se secó a temperatura constante de $40{ }^{\circ} \mathrm{C}$ durante 72 horas, posteriormente se pulverizó y se almacenó en bolsas de plástico con cierre hermético. La obtención de los extractos se realizó por maceración pasiva utilizando etanol y hexano como solventes, para esto $150 \mathrm{~g}$ del material vegetal pulverizado se sumergieron en $400 \mathrm{~mL}$ de cada solvente por separado durante 72 horas en oscuridad total. Para la obtención del extracto acuoso el material vegetal se llevó a decocción manteniendo temperatura de ebullición por 20 minutos. La concentración de los extractos obtenidos se realizó por destilación a $78{ }^{\circ} \mathrm{C}$ y $68{ }^{\circ} \mathrm{C}$ para etanol y hexano respectivamente, así mismo el extracto acuoso se mantuvo en decocción a temperatura de ebullición durante tres horas hasta evaporar la mayor cantidad de agua posible. Los extractos fueron conservados en frascos de vidrio ámbar en refrigeración hasta su uso. Las concentraciones de aplicación fueron 7, 5 y $2 \%$ pv de extracto húmedo disuelto en agua destillada.

Ensayos de germinación. Para la germinación de las semillas de $M$. pudica, se usó como sustrato papel filtro en cajas de germinación de $9 \mathrm{~cm}$ de diámetro, en cada una de ellas se depositaron 50 semillas y se aplicó un volumen de
6,0 $\mathrm{ml}$ de la solución correspondiente a cada tratamiento por caja, de acuerdo al diseño de experimentos representado en la Tabla 1, sólo una vez al comienzo de cada prueba, esto para evitar el efecto aditivo del potencial osmótico (Rodríguez, Sosa, Fernández, Reale y Villarreal, 2007). El tratamiento T1 corresponde al control positivo (agua destilada).

Se hicieron recuentos diarios, por un periodo de 12 días (Souza, Gurgel, Queiroz, Santos, 2010), y se eliminaron las semillas germinadas; se consideraron germinadas las semillas que presentaron raíz primaria igual o mayor de $2,0 \mathrm{~mm}$ de acuerdo con Juntila, 1976; Duran y Tortosa, 1985.

Para mantener la misma concentración del extracto crudo, la cantidad de agua agregada se determinó así: peso total inicial (Pi) (peso de la caja + peso del papel + peso de las semillas + peso de las soluciones), menos el peso de las semillas descartadas (PSD), menos el peso medido después de descartar las semillas (Pf) como se ve en la Ecuación 1. La cantidad de agua a aplicar (A) se sumó al valor del peso medido después de descartar las semillas $(\mathrm{Pf})$ y el resultado se utiliza como peso total inicial $(\mathrm{Pi})$ de la siguiente medida (Ecuación 2).

$$
\begin{gathered}
A=P i-P S D-P f \\
P i=A+P f
\end{gathered}
$$

$\mathrm{Al}$ tratamiento que presentó mayor porcentaje de inhibición de germinación, se le realizaron pruebas preliminares que indicarían la posible presencia de grupos de metabolitos secundarios como son: alcaloides saponinas, triterpenos, taninos flavonoides, glucósidos cianogénicos; las pruebas se

\begin{tabular}{|c|c|c|}
\hline $\begin{array}{c}\text { Factor } A \\
\text { Parte de la planta }\end{array}$ & $\begin{array}{l}\text { Factor B } \\
\text { Solvente }\end{array}$ & $\begin{array}{c}\text { Factor C } \\
\text { Concentración \% } \% \text { v }\end{array}$ \\
\hline \multirow{3}{*}{ Hojas } & Etanol & $\begin{array}{c}\text { Control } \\
7 \\
5 \\
2\end{array}$ \\
\hline & Hexano & $\begin{array}{c}\text { Control } \\
7 \\
5 \\
2\end{array}$ \\
\hline & Agua & $\begin{array}{c}\text { Control } \\
7 \\
5 \\
2\end{array}$ \\
\hline \multirow{3}{*}{ Corteza } & Etanol & $\begin{array}{c}\text { Control } \\
7 \\
5 \\
2\end{array}$ \\
\hline & Hexano & $\begin{array}{c}\text { Control } \\
7 \\
5 \\
2\end{array}$ \\
\hline & Agua & $\begin{array}{c}\text { Control } \\
7 \\
5 \\
2\end{array}$ \\
\hline
\end{tabular}

Tabla 1. Representación esquemática del diseño experimental. 
realizaron utilizando los protocolos fitoquímicos descritos por Barba, (1997), Domínguez, (1989), Harborne, (1989), Farnsworth y Segelmen, (1999), Rodas, (2004).

Diseño experimental. Para analizar el efecto de los factores se utilizó un diseño Multi-factor categórico con tres replicas, Tabla 1; diseño experimental que estimó los efectos de 3 factores categóricos. El factor A (Parte de la planta) cuenta con dos niveles (hojas y corteza), el factor $\mathrm{b}$ (Solvente) cuenta con tres niveles (etanol, hexano y medio acuoso) él factor c (Concentración del extracto) cuenta con cuatro niveles ( 7,5 y $2 \%$ pv y control). los datos fueron analizados utilizando el programa Statgraphics Centurion XVI (Stat Point Technologies Inc.).

La unidad experimental fue 50 semillas de M. pudica y la variable respuesta fue porcentaje de semillas germinadas expresada en decimales. Para analizar los efectos de los tratamientos sobre la variable respuesta se realizó una ANOVA simple al $95 \%$ de significancia y para determinar las diferencias significativas entre las medias se realizó una prueba de rangos múltiples con HSD TUKEY al 95\%, se tomaron los contrastes que miden el tratamiento control frente a los otros tratamientos. Se utilizó agua destilada para el tratamiento control.

\section{Resultados y discusión}

Rendimiento del extracto. La cantidad de extracto obtenido varió dependiendo del solvente y de la parte de la planta de donde se extrajo, el mayor rendimiento se obtuvo utilizando etanol como solvente y hojas como parte de la planta, por otro lado, la cantidad de extracto más bajo se obtuvo con hexano-corteza seguido de hexano-hojas, mientras que, aguahojas y agua-corteza tuvieron un rendimiento medio.

Efectos de niveles de factores en el porcentaje de germinación. Los resultados obtenidos al final del bioensayo se analizaron con STATGRAPHICS bajo el diseño multifactorial categórico, los datos se transformaron por ASIN

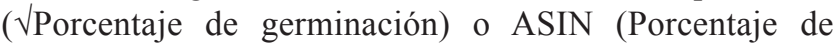
germinación $(\wedge 1 / 2)$, que corresponde a la trasformación utilizada para normalizar los datos para el análisis de ANOVA. Además el control se introdujo como un nivel más del factor $\mathrm{C}$ (Concentracion)

Así mismo se establecieron las hipótesis nulas de igualdad de medias para todos los niveles de los factores experimentales que indicarían que no hay diferencias entre las medias de los factores, así:

$$
H_{\mathrm{o}}: \mu_{A 1}=\mu_{A 2}
$$

Igualdad de las medias de los dos niveles del factor $\mathrm{A}$ (Parte de la planta)

$$
H_{\mathrm{o}}: \mu_{B 1}=\mu_{A}=\mu_{B 3}
$$

Igualdad de medias de los tres niveles del factor B (Solvente)

$$
H_{0}: \mu_{C 1}=\mu_{C 2} ; \mu_{C 3}=\mu_{C 4}:
$$

Igualdad de medias para los cuatro niveles del factor $\mathrm{C}$ (Concentracion).
De igual manera se establecieron las hipótesis alternas de diferencias de medias para todos los niveles de los factores experimentales, que indicarían que si existen diferencias entre las medias de los factores.

$$
H_{\mathrm{I}}: \mu_{A 1} \neq \mu_{A 2} \text {; }
$$

Diferencia de medias de los dos niveles del factor A (Parte de la planta)

$$
H_{0}: \mu_{B 1} \neq \mu_{A} \neq \mu_{B 3}
$$

Diferencia de medias de los tres niveles del factor B (Solvente)

$$
H_{\mathrm{I}}: \mu_{C 1} \neq \mu_{C 2} ; \mu_{C 3} \neq \mu_{C 4}
$$

Diferencia de medias para los cuatro niveles del factor $\mathrm{C}$ (Concentracion)

Concentración. El tratamiento que presentó menor porcentaje de germinación, fue el extracto acuoso al $7 \%$ pv y también etanol-corteza, mientras que las semillas con germinación fueron las tratadas con los extractos con hexano o no y/o control, este parámetro estuvo determinado por la media de cada nivel de los factores (prueba de Tukey).

Análisis de varianza. El análisis de varianza que se muestra en la Tabla 2, presenta diferencia significativa para cinco grupos, los cuales son: dentro de los efectos principales, la parte de la planta, solvente y concentración y dentro de interacciones, las combinaciones parte de la planta-solvente y solvente-concentración. Por lo tanto se rechazan las hipótesis nulas (Ho) de igualdad de medias y se aceptan las hipótesis alternativas (Ha).

Todas las razones-F se basan en el cuadrado medio del error residual.

La Tabla ANOVA descompone la variabilidad de ASIN

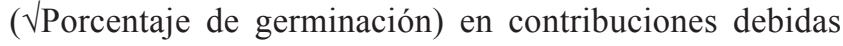
a varios factores. Puesto que se ha escogido la suma de cuadrados Tipo III (por omisión), la contribución de cada factor se mide eliminando los efectos de los demás factores. Los valores-P prueban la significancia estadística de cada uno de los factores. Puesto que 5 valores-P son menores que 0,05 , estos factores tienen un efecto estadísticamente significativo

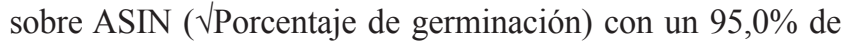
nivel de confianza.

Tabla 2. Análisis de Varianza para ASIN ( $\sqrt{\text { PORCENTAJE DE }}$ GERMINACIÓN) - Suma de Cuadrados Tipo III

\begin{tabular}{lcrcrc}
\hline Fuente & $\begin{array}{c}\text { Suma de } \\
\text { Cuadrados }\end{array}$ & $\begin{array}{c}\text { Gl } \\
\text { Cuadrado } \\
\text { Medio }\end{array}$ & Razón-F & Valor-P \\
\hline Efectos principales & & & & & \\
\hline A:parte de la planta & 385,158 & 1 & 385,158 & 4,23 & $\mathbf{0 , 0 4 5 3}$ \\
B:solvente & 4163,9 & 2 & 2081,95 & 22,84 & $\mathbf{0}$ \\
C:concentracion & 7118,27 & 3 & 2372,76 & 26,03 & $\mathbf{0}$ \\
Interacciones & & & & & \\
AB & 1541,54 & 2 & 770,771 & 8,46 & $\mathbf{0 , 0 0 0 7}$ \\
AC & 155,503 & 3 & 51,8344 & 0,57 & 0,6384 \\
BC & 4909,65 & 6 & 818,276 & 8,98 & $\mathbf{0}$ \\
ABC & 760,861 & 6 & 126,81 & 1,39 & 0,2375 \\
Residuos & 4375,3 & 48 & 91,1521 & & \\
Total (corregido) & 23410,2 & 71 & & & \\
\hline
\end{tabular}


El análisis de varianza mostró una diferencia significativa para cinco grupos, los cuales son: Los efectos principales, la parte de la planta, solvente y concentración y dentro de interacciones, las combinaciones parte de la planta-solvente y solvente-concentración. (Esto denota una relación directa para la mejor respuesta)

Para el contraste factor A (parte de la planta) cortezahojas, existe diferencia estadísticamente significativa, esto nos indica que, en este caso, los extractos de corteza tuvieron un menor número de semillas germinadas, por lo tanto, mayor actividad alelopática vs los extractos de hojas, estadísticamente con un nivel de confianza del 95\%.

En comparación, Souza, et al. (2010), observaron que los extractos de corteza y hojas de Copaifera duckei, poseen bajo potencial para inhibir la germinación de $M$. pudica, también señalan que, para la especie Copaifera martii y Copaifera reticulata el extracto de hojas presento inhibición alrededor de $60 \%$ y 50\%, respectivamente. De las tres especies, sólo los extractos de corteza de $C$. reticulada mostraron ser eficaces en la inhibición de la germinación de las semillas de $M$. pudica, esto contrasta con los resultados para C. pubiflora del presente estudio donde la corteza tuvo un mejor desempeño para inhibir la germinación de $M$. pudica.

Por otro lado, los resultados de contraste posibles con los diferentes niveles del factor B (Solvente); dos contrastes, etanol-hexano y hexano-medio acuoso, poseen diferencia estadísticamente significativas, también podemos observar un grupo homogéneo entre etanol y medio acuoso, por lo tanto, si comparamos etanol y medio acuoso frente a hexano, este último tendrá un mayor porcentaje de germinación, esto nos indica que, los extractos hexánicos no tienen efectos significativos de actividad alelopática, mientras los extractos acuosos y etanólicos sí.

En este sentido Souza, et al. (2010) indican que C. duckey, no presenta actividad alelopática con ningún extracto, mientras que para C. martii, y para C. reticulata el extracto etanolico es un $70 \%$ más efectivo que el extracto hexánico, lo que concuerda con el presente estudio.

Las medias de los niveles del factor B (solvente), aparte de lo mencionado anteriormente, permiten observar que, aunque el contraste etanol-medio acuoso no presenta diferencias estadísticamente significativas, el extracto etanólico tiene un menor porcentaje de germinación, frente a los extractos acuosos, lo que indicaría una mayor actividad alelopática sin que sea significativa.

Los contrastes entre los niveles del factor $\mathrm{C}$ (concentración), permiten determinar que no existen diferencias significativas entre las concentraciones $7,5,2 \% \mathrm{pv}$ de los extractos, pero si diferencias significativas, frente al control, por lo tanto, los extractos brutos, en cualquier concentración, tienen efectos significativos en la inhibición de la germinación, es decir en cualquier concentración de las evaluadas los extractos de C. pubiflora ejercen actividad alelopática sobre la germinación de $M$. pudica.
Ahora, cuando se relacionó el contraste para el factor anterior (solvente), con este para concentración, se pudo decir que el nivel hexano en cualquier nivel de concentración (7, 5, $2 \% \mathrm{pv}$, control), no tuvieron efectos significativos sobre el porcentaje de germinación, en cambio el extracto etanólico y el extracto acuoso sí; por lo tanto, los extractos etanólicos y acuosos de C. pubiflora en cualquier concentración de las evaluadas presentan actividad alelopática sobre la germinación de M. pudica.

Por otro lado, también pudimos, deducir que, aunque no sean significativas las diferencias, a mayor concentración, mayor es el efecto inhibitorio de la germinación, en consecuencia, los mejores resultados de actividad alelopática de los extractos, se obtienen con la concentración más alta evaluada, $7 \%$ pv.

Efectos de las interacciones de los factores. Para analizar los efectos de las interacciones que de acuerdo con la ANOVA, tienen diferencias estadísticamente significativas, se utilizaron los gráficos de interacción, que mostraban la media de cada combinación del factor A (parte de la planta) y el factor B (solvente); esto demostró como el efecto del solvente etanol es significativamente más fuerte en combinación con corteza, mientras que en hojas tiene un efecto, estadísticamente similar al solvente Hexano y Acuoso, los cuales al mismo tiempo tienen un efecto similar en hojas y corteza.

La Figura 1 describe el comportamiento de las medias de los niveles del factor B (solvente) combinado con los niveles del factor C (concentración), el efecto que más se puede señalar es que, el nivel Hexano tiene en todos los niveles de concentración $(7,5,2 \% \mathrm{pv}$, y control) la misma fuerza, por lo tanto, se puede asegurar que el extracto hexánico en todas las concentraciones evaluadas, es estadísticamente similar al control, entonces, el efecto alelopático de los extractos hexánicos no es estadísticamente influyente en la germinación de $M$. pudica.

Así mismo, pudimos determinar que cualquier nivel de concentración, tiene una fuerza estadísticamente igual en etanol y medio acuoso. Además las concentraciones $7 \%, 5 \%, 2 \%$, de etanol y Medio acuoso afectan con mayor potencia la germinación de $M$. pudica, frente a control. Por consiguiente el extracto etanolico y acuoso de C. pubiflora en cualquier concentración evaluada, presenta actividad alelopática sobre la germinación de $M$. pudica.

La Figura 2 muestra la comparación de todos los tratamientos frente al tratamiento control; estos indicaron que existen diferencias significativas para 10 tratamientos (T2, T3, T8, T9, T11, T12, T13, T17, T18, T19) es decir el porcentaje de germinación se ve significativamente afectado, en comparación con el control, por lo tanto tienen algún nivel de actividad alelopática en la germinación de $M$. pudica. La grafica también muestra la diferencia; entre mayor sea la diferencia mayor es el efecto sobre el porcentaje de germinación. El mejor tratamiento es el T11 (corteza*etanol*7\%). 


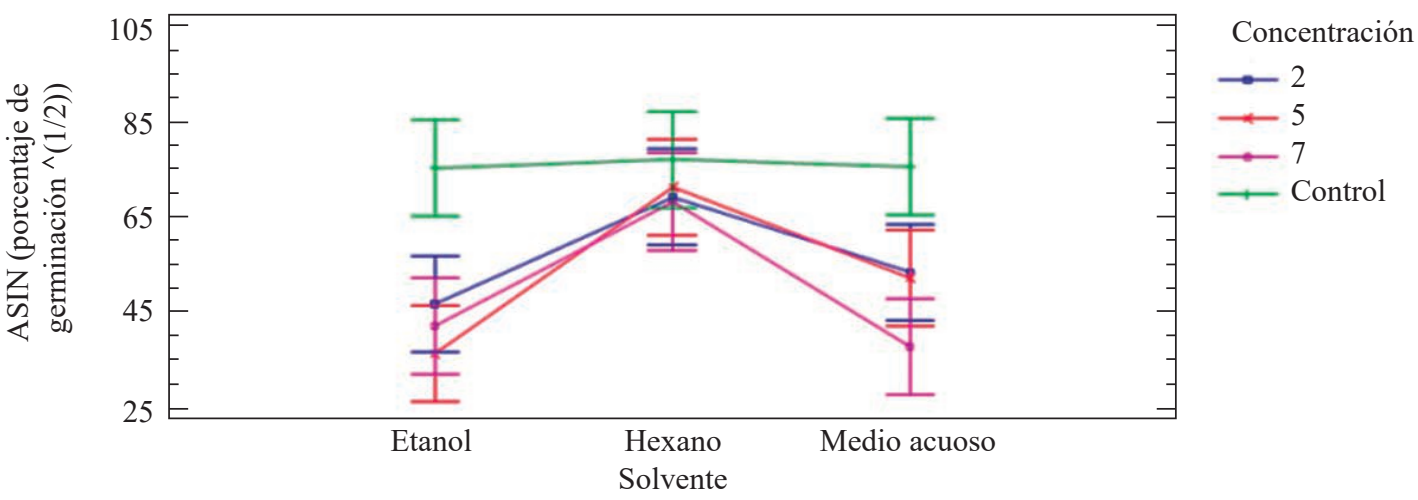

Figura 1. Interacción para solvente*concentración. HSD Tukey 95\%

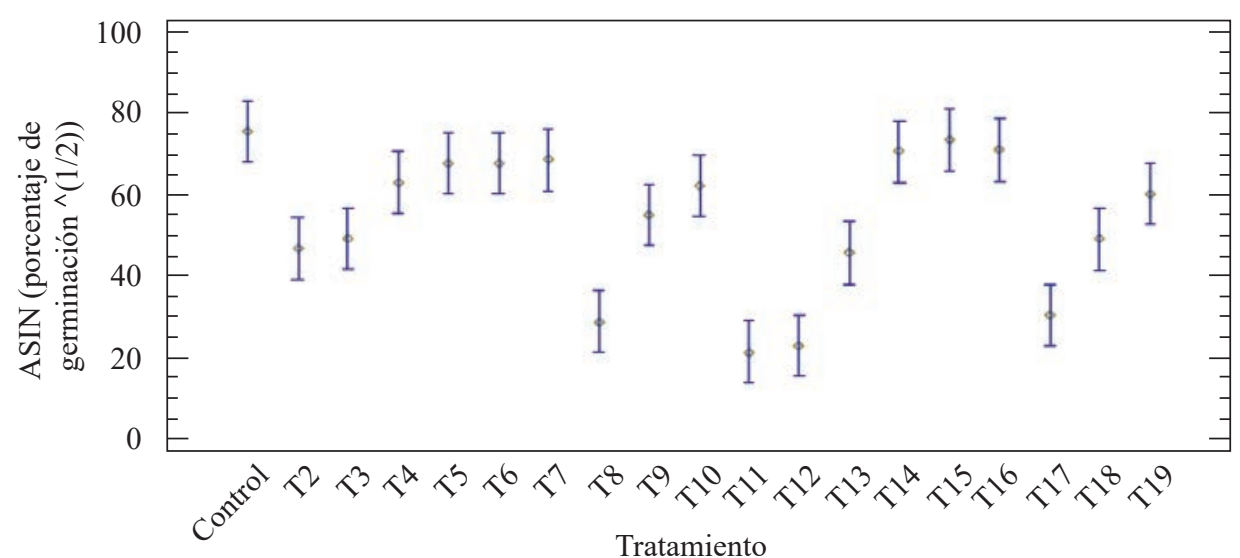

Figura 2. Medias de los tratamientos HSD Tukey $95 \%$

Los contrastes con menor diferencia respecto al Control, fueron T5, T6, T7, T14, T15, T16, estos corresponden a tratamientos en donde el solvente fue hexano, de allí se puede asegurar que el extracto hexánico de C. pubiflora, aplicado no presenta actividad alelopática sobre la germinación de M. pudica.

Caracterización preliminar. Las pruebas preliminares de identificación de metabolitos secundarios (Tabla 3) realizadas al extracto etanólico de corteza de C. pubiflora indican la presencia de compuestos reportados con alguna actividad en la defensa y adaptación de la planta frente a factores de estrés ambiental, esta respuesta requiere de una serie de procesos metabólicos dentro de los que se activa la biosíntesis de metabolitos secundarios, los cuales actúan en asociación.

En el estudio realizado por Corpoica, (2003), se identificó la presencia activa de flavonoides en los extractos etanólicos de plántulas de Rumex crispus, especie que presenta alta actividad alelopática, los datos se tomaron en tres estados de crecimiento lo que permitió confirmar la presencia constante de flavonoides, terpenos, sesquiterpenos lactonas, quinonas, glucósidos y cumarinas, pero no de alcaloides. Esto puede indicar que los flavonoides y glucósidos encontrados en C. pubiflora, intervienen en la inhibición de la germinación de $M$. pudica.

Los flavonoides son agentes alelopáticos reconocidos, dentro de estos se encuentra el flavonoide kulkulkanin, que afecta significativamente la germinación Izasa, Gimenez,

Tabla 3. Resumen de pruebas preliminares de identificación de metabolitos por caracterización fitoquímica

\begin{tabular}{|c|c|}
\hline Metabolito & Resultado de la prueba \\
\hline Taninos ${ }^{\text {a, b }}$ & Positivo para taninos catéquicos \\
\hline Compuestos reductores ${ }^{\mathrm{c}, \mathrm{d} \mathrm{ye}}$ & Positivo \\
\hline Aminas cuaternarias cy d. & Positivo \\
\hline Flavofenos ${ }^{\mathrm{c}, \mathrm{dye}}$ & Positivo \\
\hline Chalconas ${ }^{\mathrm{c}, \mathrm{dye}}$ & Positivo \\
\hline Triterpenos ${ }^{\text {y } b .}$ & Positivo \\
\hline Flavonoides ${ }^{\mathrm{b}}$ & Positivo \\
\hline Leucoantocianinas ${ }^{c y d}$ & Negativo \\
\hline Antocianinas ${ }^{\mathrm{cyd}}$ & Positivo \\
\hline Alcaloides ${ }^{\text {fyf }}$ & Negativo \\
\hline Saponinas $f, y g$ & Negativo \\
\hline Glucósidos cianogenicos ${ }^{\text {b, fyg }}$ & Positivo \\
\hline
\end{tabular}

Referentes: a: Trase (1986); b: Barba, (1997); c: Cruz, (1995); d: Tally,

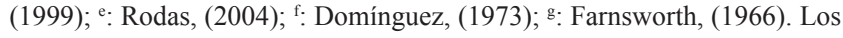
referentes se tomaron como protocolos para la realización de las pruebas de reconocimiento de metabolitos. 
Galvan y Restrepo (2007). Otro grupo de metabolitos relacionados con efectos alelopáticos para la germinación de tomate y lechuga son los taninos catequicos o condensados (Silva \& Santos 2010), sin embargo, hay que recordar que los aleloquímicos pueden ser muy específicos con la especie receptora.

Por otro lado, los triterpenos podrían no tener efecto aleloquímico, (Luz, Filho, Guilohn, y Vilhena, 2010) la evaluación de los triterpenos: lupenona, lupeol, e hidroxilupeno, obtenidos a partir de las hojas de Acacia mangium, a diferentes $\mathrm{pH}$, los cuales no mostraron ningún efecto inhibitorio de la germinación de $M$. pudica. Sin embargo algunos monoterpenos sí ejercen algún tipo de alelopatía. Un grupo de metabolitos asociado también a la actividad alelopática es el de los glucósidos cianogénicos que producen ácido cianhídrico que puede actuar como metabolito de inducción de la respuesta de defensa en plantas (Putnam, 1995).

\section{Conclusiones}

El estudio del efecto alelopático de extractos obtenidos a partir de diferentes órganos de Copaifera pubiflora sobre la germinación de Mimosa pudica, permite establecer que la fracción etanólica de corteza y tallo presenta actividad inhibitoria en las diferentes concentraciones aplicadas, sin embargo se pudo observar que el extracto de corteza a concentración de $7 \%$ pv alcanzó un porcentaje de inhibición de la germinación de $87 \%$, valor más alto para este trabajo, con esto se comprueba que los extractos naturales requieren de concentraciones altas para potenciar su actividad biológica, siendo esta una de las principales desventajas de su uso en agricultura, de igual manera estos resultados indican que existe una distribución de los metabolitos secundarios en la planta a nivel de órganos, lo que influye en la efectividad de los extractos obtenidos. Los extractos acuosos de hojas y tallo también presentan actividad inhibitoria de la germinación en las tres concentraciones de aplicación evaluadas, mientras los extractos hexánicos de los dos órganos utilizados no tuvieron efecto sobre la germinación en comparación con el tratamiento control, por lo tanto se asocia el efecto de inhibición a los metabolitos secundarios de alta polaridad como flavonoides, taninos, glucósidos cianogénicos, que de manera preliminar se identificaron para este especie.

\section{Agradecimientos}

Los autores desean expresar sus agradecimientos al doctor José Alfredo Camargo Martínez por su apoyo en la edición final del manuscrito.

\section{Conflicto de intereses}

Los autores manifiestan que no tienen conflicto de intereses.

\section{Referencias}

Acero, L. E. 2005. Plantas útiles de la cuenca del Orinoco. Ecopetrol. BP. Corporinoquia. Editorial. Asociación Santiago de Las Atalayas, Corporinoquia, Bogotá.
Abad, A., Tiscareño, M., Villar, C \& Varela, S. 2012. Manejo de maleza en cultivos tropicales. Universidad Autónoma de San Luis Potosí. Universidad Autónoma de Tamaulipas. México D. F.

Ariza, C. A., \& Merchán, P. J. A. 2012. Identificación y clasificación en biotipos de las malezas asociadas con el cultivo de la palma de aceite. Revista Ciencia y Agricultura, 9 (2): 87-96.

Barba, C. J. 1997. Introducción al Laboratorio de Fitoquímica. México D. F.: Universidad Autónoma Metropolitana.

Bernal, H., Martínez, H., \& Sánchez, G. 2011. Pautas para el conocimiento, conservación y uso sostenible de las plantas medicinales nativas en Colombia: estrategia nacional para la conservación de plantas. Ministerio de Ambiente, Vivienda y Desarrollo Territorial.

Blanco, Y. 2013. Revisión bibliográfica. La utilización de la alelopatía y sus efectos en diferentes cultivos agrícolas. Cultivos tropicales, 27 (3): 5-16.

Cascon, V., \& Gilbert, B. 2000. Characterization of the chemical composition of oleoresins of Copaifera guianensis Desf., Copaifera duckei Dwyer and Copaifera multijuga Hayne. Phytochemistry, 55 (7): 773-778.

CORPOICA. 2003. contenían varias sustancias alelopáticas: flavonoides, 5-deoxiflavonas, 7-8- dihidroflavononas. Revista CORPOICA, 1-2.

Cruz, L. 1995. Guía práctica de productos naturales y fitoquímica, (1 ed.). Guatemala: Universitaria.

CUNHA, A. G. 1999. Dicionário histórico das palavras portuguesas de origem tupi. Brasília: Melhoramentos. 120 p.

Cutié, A., Rosales, R., \& Gámez, R. 2015. Mimosa pudica: una modalidad local de sustancia de abuso. MEDISAN, 19 (12): $1556-1560$.

Decker, B. L. 1986. World geodetic system 1984. Defense Mapping Agency Aerospace Center St Louis Afs Mo.

Domínguez, X. 1989. Métodos de Investigación fitoquímica. México D. F.: Limusa.

Duran, JM; Tortosa, ME. 1985. El efecto de la escarificación mecánica y química en la germinación de chalock (Sinapisarvensis L.). Seed Sci. Technol., 13 (1): 155-163.

Gurgel, E. S. C. 2009. Morfoanatomia, perfil químico e atividade alelopática de três espécies de Copaifera L.(Leguminosae Caesalpinioideae) nativas da amazônia.

Hoyos, V., Martínez, M. J., \& Plaza, G. 2016. Malezas asociadas a los cultivos de cítricos, guayaba, maracuyá y piña en el departamento del Meta, Colombia.Revista Colombiana de Ciencias Hortícolas, 9 (2): 247-258.

Farnsworth, N., \& Segelmen, A. 1999. Biological and Phytochemical Evaluation of plants. J. Pharm Sci.

Franco, D. M. 2013. Atividade alelopática de Copaifera langsdorffii DESF: abordagem fitoquímica e molecular.

Ferreira, A. B. H. 1988. Novo dicionário da língua portuguesa. Rio de Janeiro: Nova Fronteira, 1498 p.

Harborne, J. 1989. Phytochemical Methods. (segunda ed.). Chapman and Hall. INSTITUTE., S. 1989. Statistical Analysis System. User's guide. Version 6.4. ed. Cary: 1989. 846 p.

Izasa, J., Gimenez, F., Galvan, J. I., \& Restrepo, J. C. 2007. Actividad alelopática de algunas especies de los géneros miconia, tibouchina, henriettella, tococa, aciotis y bellucia (Melastomataceae). Scientia et Technica A, 409-413. 
Juntila, O. 1976. semillas y la germinación de embriones en S. vulgaris y $\mathrm{S}$. reflejo de los efectos de la temperatura durante el desarrollo de la semilla. Physiol. Plant., 29: 264-268.

Labouriao, L. G. 1983. A germinação de sementes. Washington: OEA: 170

Lewis, G., Schrire, B., MacKinder, B., \& Lock, M. 2005. Legumes of the world Royal Botanical Gardens. Kew, UK.

Luz S, S. F. 2010. Atividade alelopática de substâncias químicas isoladas da Acacia mangium e suas variações em função do PH. Planta daninha vol. 28 no. 3, 1-5

Mehhboob, N.; Saleem, B. y Qureshi, M. J. Plant and chemical environment. Pakistan Journal of Biological-Sciences, 2000, vol. 3, no. 8, p. 1305-1307, 2000

Menza, H. D., \& Salazar, L. F. 2006. Resistencia de Eleusine indica al glifosato en cafetales de la zona cafetera central de Colombia.

Martins-da-Silva, R. C. V. 2006. Taxonomia das espécies de Copaifera L. (Leguminosae Caesalpinoideae) ocorrentes na Amazônia Brasileira. 2006. 258 f. Tese (Doutorado em Botância) - Universidade Federal do Rio de Janeiro, Rio de Janeiro.

Narwal, S. S., \& Haouala, R. 2011. Allelopathic strategies for ecological weed management. Herbologia, 12 (1): 29-56.

Oliveros-Bastidas, A. D. J. 2008. El fenómeno alelopático. El concepto, las estrategias de estudio y su aplicación en la búsqueda de herbicidas naturales. Revista QuímicaViva, 7 (1).

Ospina Ante, A., Beer, J., Kapp, G., Lucas, C., Vargas, F., A Jiménez, F., \& Schlönvoigt, A. 2003. Agroforestería: aportes conceptuales, metodológicos y prácticos para el estudio agroforestal (No. 631.58 O83). Asociación del Colectivo de Agroecología del Suroccidentale Colombiano, Cali (Colombia).

Ortiz H., Sánchez W., Méndez A. \& Murillo E. 2009: Potencial antioxidante de hojas y corteza de Bauhinia kalbreyeri Harms: Contribución de sus flavonoides en esta actividad. Rev. Acad. Colomb. Cienc. 33 (127): 183-191, 2009. ISSN 0370-3908.
Putnam, A. R. 1995. Weed allelopathy. En S. O. Duke, Reproduction and Ecophysiology (págs. 131-155). Boca Raton: Duke, S. O.

Ramirez, N., \& Arroyo, M. K. 1990. Estructura poblacional de Copaifera pubiflora Benth. (Leguminosae; Caesalpinioideae) en los Altos Llanos Centrales de Venezuela. Biotropica, 124-132.

Rodas, I. M. 2004. Identificación de familias de metabolitos secundarios en Myrica cerifera. Guatemala: Universidad de San Carlos de Guatemala.

Rodríguez Rivera, M. F., Sosa, L. R., Fernández, E. A., Reale, M. I., \& Villarreal, V. 2007. Efecto del estrés hídrico a distintas temperaturas sobre la germinación de semillas de Bulnesia retama (Gill. ex. Hook.) Griseb.-Zigofiláceas-en San Luis, Argentina. Phyton (Buenos Aires), 76: 5-17

Souza Filho, A., Gurgel, E., Queiroz, M., \& Santos, J. 2010. Atividade alelopática de extratos brutos de três espécies de copaifera (leguminosaecaesalpinioideae). Planta daninha, 28 (4): 746-747.

Society, I. A. 1996. International Allelopathy Society. Recuperado el 02 de 09 de 2014, de International Allelopathy Society: http://www.as.uca.es/bylaws.htm

STATGRAPHICS. 2006. Diseño de Experimentos - Diseños para Varios Factores Categóricos. Rev, 1-8.

Tally, W. 1999. Seminario taller mesoamericano, metabolitos de interés nutricional en plantas comestibles de la región. Guatemala: Universidad San Carlos de Guatemala. 26 al 30 de abril.

Zamorano, C. 2006. Alelopatía: un nuevo reto en la ciencia de las arvenses en el trópico. Agron, 14 (1): 7-15. 